\title{
Nonlinear Oscillation of the Magnetosphere around Neutron Stars
}

\author{
Hi toshi HANAMI \\ Department of Physics, Hokkaido University \\ Sapporo 060, JAPAN.
}

\begin{abstract}
SUMMARY; We investigate the unsteady motion of mass reservoir formed by the accretion onto the magnetosphere around rotating neutron stars. The unsteady motion of the reservoir induces secondary accretion to neutron star by $R-T$ instability. The nonperiodic or quasiperiodic phenomena of $X$-ray bursters seems to be related to this property of mass reservoir on the magnetosphere. We classify the typical dynamical state of the reservoir into three types with the parameters which are accretion rate $\dot{M}$ and angular velocity of neutron star $\Omega$. They are nonsequential oscillatiof, sequential periodic (quasi-periodic) oscillation, and chatic oscillation states.
\end{abstract}

1. Physical Model; We propose a symplified model for the non-linear-like phenomena in X-ray sources, considering the properties of Rayleigh-Taylor instability on the magnetopause which is formed by the accreting matter to the neutron star, and then obtain the motion of this magnetopause,

We assume that the stellar magnetic field is dipolar ( $\alpha \dot{\sim}^{-3}$ ), and has axial symmetry everywhere. We use cylindrical coordinates $(\tilde{\omega}, \Phi, z)$ centered on the neutron star and aligned with the stellar rotation axis. This configuration is sketched in Figure 1. We obtain the nondimensionalized equations which construct a complete set for the dynamics of reservoir ring, as following,

$$
\begin{aligned}
& \Sigma \frac{d V}{d t}=Q\left(B p^{2}-\alpha\right) x+1 / 2 \cdot\left(-x^{-2}+2 H^{2} x^{-3}\right)-\left(V x^{(d+1)}+\dot{\Sigma}_{a c c}\right)^{2} \cdot x^{-(d+1)}+(1), \frac{d x}{d t}=V \leftarrow(2), \\
& \Sigma \frac{d H}{d t}=2 Q x^{2} \cdot B p \cdot B t+\dot{\Sigma}_{i n}(x / 2)^{1 / 2}+(3), \frac{d B t}{d t}=\frac{B p}{L}(H / x-Q x)-\dot{\Sigma}_{10 s s} / \Sigma \cdot B t \leftarrow(4), \\
& \frac{d \Sigma}{d t}=\dot{\Sigma}_{\text {in }}-\dot{\Sigma}_{\text {loss }}+(5), \dot{\Sigma}_{\text {in }}=\theta\left(V x^{(d+1)}+\dot{\Sigma}_{a c c}\right) \leftarrow(6), \dot{\Sigma}_{\text {loss }}=\Sigma \cdot\left(\theta\left(\frac{d V}{d t} / x\right) f^{1 / 2} \leftarrow(7),\right.
\end{aligned}
$$

$B p=x^{-3} \leftarrow(8)$, where $t, x, v, H, B p, B t, \Sigma, \dot{\Sigma}$, $\Omega$, and $\dot{\Sigma}$ are the nondimensinal time, radius, radial velocity, specifiçangular momenless, mass, poloidal, toroidal magnetic field of the reservoir, accretion rate, angular velocity of central star, and mass loss rate from reservoir.

Figure

initial condition fixed on $\Sigma=1.0, V=0.0, x=1.0$,

$H=0.1, B t=0.0$, and the various values of the control parameters $\dot{\Sigma}$ and $\Omega$. We get

three types solutions, as shown below.

$\begin{array}{lrrl} & \Omega & \dot{E} a c c & \text { dynamics } \\ \text { case (a) } & 1.0 & 3.00 & \text { Periodic } \\ \text { case (b) } & -1.0 & 2.50 & \text { Expansion } \\ \text { case (c) } & -1.0 & 2.53 & \text { Chaotic } \\ \text { case (d) } & -1.0 & 3.00 & \text { Periodic } \\ \text { case (e) } & 0.0 & 1.00 & \text { Periodic } \\ \text { case (f) } & -1.0 & 2.80 & \text { Periodic }\end{array}$

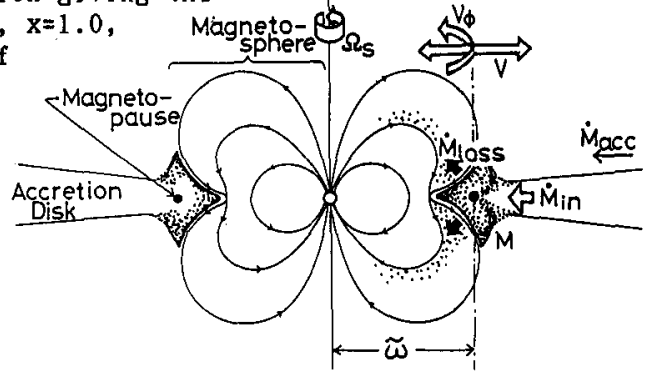

4. Physical Meanings; We will consider the physical meaning of the results shown in above section. Now, for experimental approach, we introduce a test circular ring which does not change its mass $M$ and also $\Sigma$. This test $r$ ing is initially rotating with Keplerian velocity, and then, for the interaction with stellar magnetic field, the ring losses the angular momentum. When the ring falls into the magnetosphere, the rotating velocity of the ring may become the same velocity as that of the magnetosphere. 
These situations, which represent the difference between the direct and the retrograde rotation of the star against the disk, shows schematically in figure 3 (a) and (b).

Direct Rotation;

Retrograd Rotation;

$$
\begin{aligned}
& \text { A (Stable) } \frac{\text { Periodic Oscillation }}{\text { Perturvation }} \\
& \text { B (Stable) } \downarrow \\
& \text { +Mass Change } \rightarrow \text { Chaotic Oscillation } \\
& \text { D (Stable) } \quad \text { (Shift of Stable Point) }
\end{aligned}
$$

For retrograde case, the shift of two stable equilibrium points may randumly mix the oscillation modes related two points by changing of the mass. Then, the chaotic oscillation of this system may be generated.

5. Discussion; We introduce typical time scale and burst energy of this system. $\mathrm{td}=\tilde{\omega}_{0}\left(\frac{2 \mathrm{GM}_{\mathrm{s}}}{\tilde{\omega}_{0}}\right)^{-1 / 2}=0.2\left(\frac{\tilde{w}_{0}}{10^{9} \mathrm{~cm}}\right)^{3 / 2}\left(\frac{\mathrm{M}_{\mathrm{s}}}{1 \mathrm{M}_{\odot}}\right)^{-1 / 2}(\mathrm{sec})$, $E=2.58 \times 10^{38} \cdot\left(\frac{M_{S}}{1 M_{\odot}}\right)^{1 / 2}\left(\frac{\tilde{\omega}_{S}}{10^{6} \mathrm{~cm}}\right)^{3 / 2}\left(\frac{\dot{M}_{\text {acc }}}{10^{18} \mathrm{~g} / \mathrm{s}}\right)\left(\frac{\tilde{\omega}_{0}}{10^{9} \mathrm{~cm}}\right)^{3 / 2} \Delta \tau \quad$ (erg). where $\Delta \tau$ is nondimensionalized burst interval time.

$$
\mathrm{B}=2.98 \times 10^{13} \cdot\left(\frac{\mathrm{M}_{\mathrm{s}}}{1 \mathrm{M}_{\odot}}\right)^{1 / 4}\left(\frac{\tilde{\omega}_{\mathrm{s}}}{10^{6} \mathrm{~cm}}\right)^{-3}\left(\frac{\dot{\mathrm{M}}_{\mathrm{acc}}}{10^{18} \mathrm{~g} / \mathrm{s}}\right)\left(\frac{\tilde{\omega}_{0}}{10^{9} \mathrm{~cm}}\right)^{7 / 2} \Delta \mathrm{t}^{1 / 2} \quad \text { (gauss). }
$$

From X-ray observations ( van der Klis et al. 1985), the typical frequency of quasi-periodic oscillations $\omega=20 \sim 40 \mathrm{~Hz}$. The typical period of oscillation cases is $2 \mathrm{td}$ for case (a) and $5 \mathrm{td}$ for case (b). If the typical radius of this magnetosphere is $1000 \mathrm{~km}$, typical frequency of our model is a good agreement with the observational data. This value of w requires surface magnetic field $\log (B(G))=10$. On the other hand, for Rapid Burster, the time to following burst spreads from $10 \mathrm{~s}$ to $1000 \mathrm{~s}$. These time scale requires $\tilde{\omega}>r_{0} \mathrm{~cm}$, and $\log (B(G))>15$ for explaining with our model. Furthermore, the retrograde stellar rotation against that of the disk is important for chaotic behavior of Rapid Burster for our model.

\section{References}

van der Klis et al., 1985, Nature, 317,681 .

Hanami, H. 1987, M.N.R.A.S., submitted.
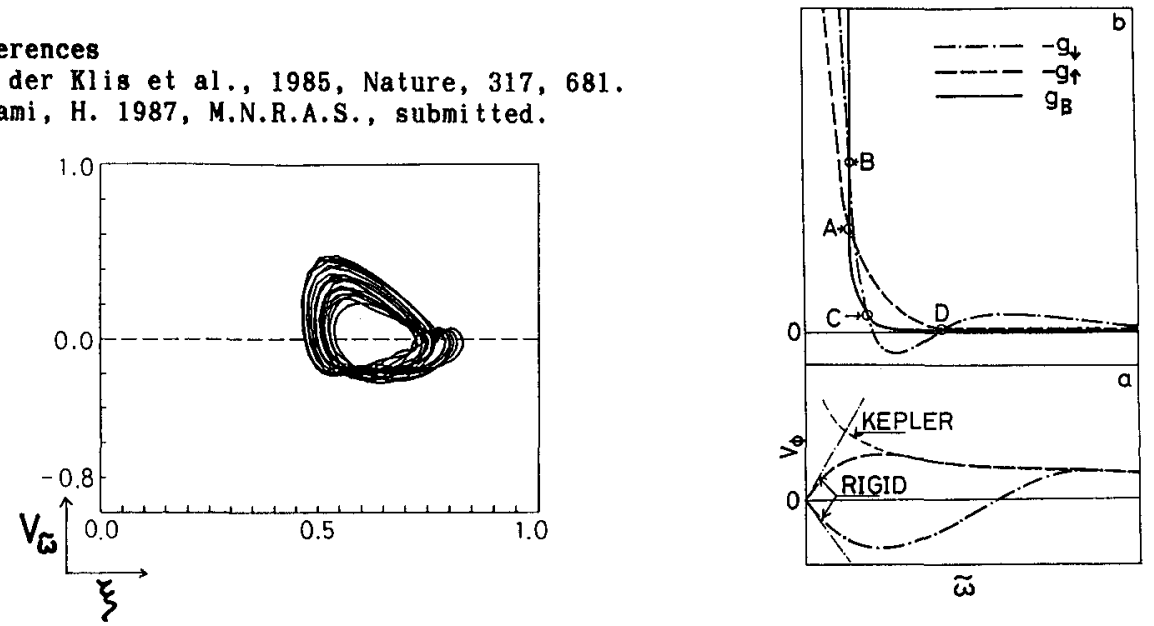

Figure 2 ;Orbits in phase space (velocity, radius) for cases (c).

Figure 3 (a); Schematic sketch of the rotation velocity as a function of the radius of test circulating ring along experimental orbit. (b);Each absolute value of the acceleration on test circular ring by magnetic pressure and effective gravity which includes the centrifugal force of rotation motion. The subscripts $\uparrow$ and $\downarrow$ of $g$ represent the values for direct and retorograd rotation cases. Open circles A, B, C, and D represent dynamical equilibrium points. 